\title{
Metabolomic profiling of gastric cancer tissues identified potential biomarkers for predicting peritoneal recurrence
}

\author{
Sanae Kaji ${ }^{1,2} \cdot$ Tomoyuki Irino $^{1} \cdot$ Masatoshi Kusuhara ${ }^{1,3} \cdot$ Rie Makuuchi $^{1} \cdot$ Yushi Yamakawa $^{1} \cdot$ Masanori Tokunaga ${ }^{1}$. \\ Yutaka Tanizawa ${ }^{1}$ - Etsuro Bando ${ }^{1} \cdot$ Taiichi Kawamura $^{1} \cdot$ Kenjiro Kami $^{4} \cdot$ Yoshiaki Ohashi ${ }^{4}$. Shun Zhang ${ }^{2}$. \\ Hajime Orita ${ }^{2} \cdot$ Hyeon-Cheol Lee-Okada ${ }^{5} \cdot$ Tetsu Fukunaga $^{2} \cdot$ Masanori Terashima $^{1}$
}

Received: 1 November 2019 / Accepted: 16 March 2020 / Published online: 26 March 2020

(c) The International Gastric Cancer Association and The Japanese Gastric Cancer Association 2020

\begin{abstract}
Background Metabolomics is useful for analyzing the nutrients necessary for cancer progression, as the proliferation is regulated by available nutrients. We studied the metabolomic profile of gastric cancer (GC) tissue to elucidate the associations between metabolism and recurrence.

Methods Cancer and adjacent non-cancerous tissues were obtained in a pair-wise manner from 140 patients with GC who underwent gastrectomy. Frozen tissues were homogenized and analyzed by capillary electrophoresis time-of-flight mass spectrometry (CE-TOFMS). Metabolites were further assessed based on the presence or absence of recurrence.

Results Ninety-three metabolites were quantified. In cancer tissues, the lactate level was significantly higher and the adenylate energy charge was lower than in non-cancerous tissues. The Asp, $\beta$-Ala, GDP, and Gly levels were significantly lower in patients with recurrence than in those without. Based on ROC analyses to determine the cut-off values of the four metabolites, patients were categorized into groups at high risk and low risk of peritoneal recurrence. Logistic regression and Cox proportional hazard analyses identified $\beta$-Ala as an independent predictor of peritoneal recurrence (hazard ratio [HR] 5.21 [95\% confidence interval 1.07-35.89], $p=0.029$ ) and an independent prognostic factor for the overall survival (HR 3.44 [95\% CI 1.65-7.14], $p<0.001)$.

Conclusions The metabolomic profiles of cancer tissues differed from those of non-cancerous tissues. In addition, four metabolites were significantly associated with recurrence in GC. $\beta$-Ala was both a significant predictor of peritoneal recurrence and a prognostic factor.
\end{abstract}

Keywords Stomach neoplasms $\cdot$ Recurrence $\cdot$ Biomarkers $\cdot$ Metabolomics $\cdot$ Beta-alanine

Electronic supplementary material The online version of this article (https://doi.org/10.1007/s10120-020-01065-5) contains supplementary material, which is available to authorized users.

Masanori Terashima

m.terashima@scchr.jp

1 Division of Gastric Surgery, Shizuoka Cancer Center, 1007, Shimonagakubo Nagaizumi-cho, Sunto-gun, Shizuoka 411-8777, Japan

2 Department of Gastroenterology and Minimally Invasive Surgery, Juntendo University Hospital, Bunkyo-ku, Tokyo 113-8431, Japan

3 Shizuoka Cancer Center Research Institute, Shimonagakubo Nagaizumi-cho, Sunto-gun, Shizuoka 411-8777, Japan

4 Human Metabolome Technologies, Inc, Tsuruoka, Yamagata 997-0052, Japan

5 Department of Biochemistry, Juntendo University Graduate School of Medicine, Bunkyo-ku, Tokyo 113-8431, Japan

\section{Introduction}

Gastric cancer (GC) is the fourth most common cancer worldwide. In general, incidence is higher in East Asia and lower in Western countries. Since the middle of the twentieth century, mortality has been decreasing in Northern America, Europe, and Japan [1-3]. Nevertheless, 47,000 people still die from gastric cancer each year in Japan. Peritoneal recurrence is estimated to be the cause of $44.43 \%$ of gastric cancer deaths in the Japanese Gastric Cancer Association (a nationwide registry) data [4].

Once detected, peritoneal recurrence is considerably difficult to cure even with cutting-edge multimodal therapies (such as systemic chemotherapy, intraperitoneal chemotherapy, and hyper-thermic intraperitoneal chemotherapy) [5] . Various radiographic modalities (CT, MRI, and PET-CT) 
and staging laparoscopy, along with lavage cytology and/or peritoneal dissemination, have improved diagnostic accuracy and made it possible to decide on appropriate treatments [6]. However, occult micro-metastases of the peritoneum, which are not found during curative gastrectomy or diagnostic staging laparoscopy, might progress after surgery. In this regard, the development of biomarkers for predicting peritoneal recurrence prior to operations for GC is anticipated, as invasive examinations such as staging laparoscopy, which is performed under general anesthesia, are currently required for the assessment of patients. Appropriate therapeutic strategies can be applied to pre-selected patients with a high risk of peritoneal recurrence, which will contribute to reducing the risk of recurrence and improve the prognosis. Recently, as a biomarker to predict long-term survival in patients with pancreatic adenocarcinoma, a high concentration of ethanolamine in tumor tissue was reported to be associated with a worse prognosis [7]. There is also great interest in identifying metabolites in noninvasive samples (saliva, blood, urine, and feces) to detect early stage disease [7, 8]. Thus, finding novel biomarkers would be of significant value for understanding the tumor microenvironment and the metabolism of cancer cell growth.

Progress in "-omics" technologies, such as genomics, transcriptomics, proteomics, metabolomics, and the combination of these technologies has greatly contributed to improving the detection, diagnosis, therapy, and prognosis of cancer [9]. Several previous studies have investigated the metabolomic differences between normal and tumor tissues in GC [10-15]. However, a few studies have focused on the role of metabolism in the peritoneal recurrence of GC, with the exception of mouse xenograft studies [16].

In this study, the metabolomic profiles of normal and tumor tissue specimens surgically resected from GC patients were assessed. As in the previous reports, the levels of lactate and total amino acids in tumor tissues were higher and the ATP/AMP ratio was lower in comparison to normal tissues, which reaffirms the Warburg effect in cancer. The total glutathione level and reduced-/oxidized-glutathione ratios in tumors were significantly higher than those in normal tissues, which possibly contributes to the homeostasis of the redox status in tumor tissues [11]. Subsequently, the profiles of tumor tissues were further evaluated based on the presence or absence of recurrence-particularly for peritoneal recurrence-using cross-validation.

\section{Methods}

\section{Tissue specimens and sample cohort}

A total of 140 patients who underwent gastrectomy for GC at the Division of Gastric Surgery, Shizuoka Cancer Center between February 2010 and March 2013 were enrolled in this study (Supplementary Fig. 1). Surgical specimens were examined and graded according to the Japanese Classification of Gastric Carcinoma [17]. This study was approved by the Institutional Review Board of Shizuoka Cancer Center. Written informed consent for the use of tissue samples and data were obtained from all patients. The information of all recruited patients was available in a prospectively recorded database. The inclusion criteria of this study were patients who underwent radical gastrectomy for gastric cancer with any $\mathrm{cT}$, any $\mathrm{cN}$, and $\mathrm{M} 0$ according to the 7th TNM classification for whom $1.0 \mathrm{~g}$ tissue pieces could be collected from the primary lesion. Among these, patients who were followed up for more than 3 years were registered. The exclusion criteria were as follows: severe hepatic dysfunction (total bilirubin $>2 \mathrm{mg} / \mathrm{dL}$, aspartate transaminase $>100 \mathrm{IU} / \mathrm{l}$, alanine transaminase $>100 \mathrm{IU} / \mathrm{l}$ ); severe renal impairment (creatinine $>2 \mathrm{mg} / \mathrm{dL}$ ); anti-hepatitis B surface antigen positivity, anti-hepatitis $\mathrm{C}$ antibody positivity, fluorescent treponemal antibody-absorption test positivity; blood coagulation disorder (hypochromia $<6 \mathrm{~g} / \mathrm{dL}$, white blood cell count $<1000 /$ $\mathrm{mm}^{3}$, platelet count $<50,000 / \mathrm{mm}^{3}$, prothrombin time ratio $>1.5$ ); severe heart failure; poorly controlled diabetes mellitus in spite of the continuous use of insulin; continuous systemic steroid or immunosuppressive drug therapy; infectious disease with indication for systemic therapy; synchronous or metachronous (within 5 years) malignancies other than carcinoma in situ or intramucosal carcinoma curatively treated with local therapy; age $<20$ years; and severe psychiatric disease.

Tumor tissues and adjacent normal tissues were surgically resected and snap-frozen in liquid nitrogen. Frozen tissues were shipped to Human Metabolome Technologies (Tsuruoka, Japan), where homogenization, metabolite extraction, and a metabolome analysis with capillary electrophoresis time-of-flight mass spectrometry (CE-TOFMS) were conducted. The remaining surgical specimens were fixed in $10 \%$ formalin neutral buffer solution and embedded in paraffin. All slides were examined by at least two pathologists and a histological diagnosis was made according to the 15th Japanese Classification of Gastric Carcinoma [17].

\section{Metabolome analysis}

Metabolomic profiles of normal and tumor tissues were analyzed by the Basic Scan package of HMT (Human Metabolome Technologies) using CE-TOFMS [18, 19]. Briefly, the analysis was performed using an Agilent CE capillary electrophoresis system equipped with an Agilent 6210 time-of-flight mass spectrometer (Agilent Technologies, Waldbronn, Germany). Systems were controlled using the Agilent G2201AA ChemStation for CE version B.03.01 software program (Agilent Technologies). The spectrometer 
scanned from $m / z, 50$ to 1000 and peaks were extracted using the Master Hands automatic integration software program (Keio University, Tsuruoka, Japan) to gain peak information including the $m / z$, peak area, and migration time (MT) [20]. Signal peaks according to isotopomers, adduct ions, and other product ions of known metabolites were excluded. The other peaks were annotated according to the HMT metabolite database based on $m / z$ values with the MTs. The areas of the annotated peaks were normalized based on internal standard levels and sample amounts to obtain relative levels of each metabolite.

\section{Patient follow-up and the diagnosis of recurrence}

Patients were routinely followed up every 3 to 6 months for up to 5 years. Patients who were followed up for more than 3 years or who were diagnosed with recurrent gastric cancer within three years of August 2016 were included in the present study. Adjuvant chemotherapy using S-1 (an oral tegafur, gimeracil, and oteracil) monotherapy or combination therapy with capecitabine and oxaliplatin was used for patients with pathological stage II or III diseases. In patients with pathological stage IV, S-1 was also used for 1 or 2 years according to the physician's decision. The median follow-up period was 61.2 months. The presence or absence of recurrence was diagnosed by imaging modalities such as computed tomography, ultrasonography, positron emission tomography, and magnetic resonance imaging. We diagnosed peritoneal recurrence based on the following findings: massive ascites with positive cytology detected by puncturing abdominal cavity; the clinical or radiological identification of an intraabdominal mass, including a mass in the pouch of Douglas; or positive histopathological results obtained at surgery in the postoperative period.

\section{Cell lines and culturing}

TMK1 cells, an invasive stomach cancer cell line, were provided by Hiroshima University (Hiroshima-shi, Hiroshima, Japan). The cells were cultured at $37{ }^{\circ} \mathrm{C}$ and $5 \% \mathrm{CO}_{2}$ in RPMI 1640 medium (Wako) supplemented with $10 \%$ fetal bovine serum and penicillin/streptomycin.

\section{Transwell migration assay}

The Transwell migration assay was performed using 24-well Transwell® permeable inserts containing polycarbonate membranes (pore size: $8 \mu \mathrm{m}$ ) of $6.5 \mathrm{~mm}$ in diameter (CLS3422; Sigma-Aldrich). TMK1 cells (620 $\mu \mathrm{l}$ $\left[2 \times 10^{4}\right.$ cells $\left./ \mathrm{ml}\right]$ in serum-free RPMI medium) were dispensed into each well of the 24-well Transwell@ plates, with or without $40 \mathrm{mM} \beta$-Ala in the lower chamber, and incubated at $37{ }^{\circ} \mathrm{C}$ for $48 \mathrm{~h}$. After incubation, the cells on the upper membrane were removed using a cotton swab. The cells moving onto the lower membrane were stained by MayGiemsa staining and manually quantified. Each experiment was performed in triplicate.

\section{Study design and statistical analysis}

The metabolite levels were assessed in tumor and normal tissues, the absence or presence of recurrence, and clinicopathological factors, using a non-parametric Mann-Whitney $U$ test with Bonferroni correction for multiple comparisons. A Mann-Whitney $U$ test was also employed with FDR correction for multiple testing with $q$-values (Benjamini-Hochberg method) to explore the metabolites associated with the absence or presence of peritoneal recurrence. $P$ values of $<0.05$ were considered to indicate statistical significance. A principal component analysis (PCA) was performed to compare the normal and tumor tissue profiles. A partial least-squares (PLS) analysis was used as a classification model for exploring metabolites that were associated with the absence or presence of peritoneal recurrence [21]. The performance in the prediction of peritoneal recurrence was assessed based on the area under the curve (AUC) of a receiver-operating characteristics (ROC) curve with cut-off values determined by the Youden index. The performance in the prediction of peritoneal recurrence was further evaluated by eightfold cross-validation. The relapse-free survival of the two groups, classified based on the cut-off values for each metabolite, was compared using Kaplan-Meier curves and the log-rank test. A logistic regression model and a Cox proportional hazard model were used to assess the performance in the prediction of peritoneal recurrence and survival analysis, respectively.

\section{Results}

\section{Patient characteristics}

Among 1078 patients who were treated at Shizuoka Cancer Center from Feb 2010 to Mar 2013, 200 patients agreed to participate in this study (Supplementary Fig. 1). Among these, we analyzed a total of 160 patients; 147 of whom underwent R0 or R1 resection. Of these, 140 patients were followed up for more than 3 years or were diagnosed with recurrent gastric cancer within 3 years. The clinicopathological characteristics of the 140 patients are summarized in Supplementary Table 1.

One hundred nine patients received $\mathrm{R} 0$ resection. The remaining 31 patients received R1 resection (microscopically positive resection margin and/or cancer cells in peritoneal cytology); peritoneal cytology was positive for cancer cells in all of these cases. Among the 140 patients, 
43 experienced recurrence (peritoneal recurrence, $n=35$; lymph-node recurrence, $n=17$; and hematogenous recurrence, $n=8$ ) within 3 years after gastric surgery.

\section{Tumor tissues vs. normal tissues}

Among the peaks detected by CE-TOFMS, 96 metabolites involved in energy metabolism, including glycolysis, pentose phosphate pathway, TCA cycle, urea cycle, amino acid, and nucleotide metabolism, were quantified. Based on the metabolite data, PCA clearly distinguished tumor tissue from normal tissue profiles along the PC1 axis (Fig. 1). The



Fig. 1 Principal component analysis of normal and tumor tissues (red and blue marks, respectively) levels of 58 metabolites were significantly different between tumor and normal tissues. Tumor tissues contained significantly higher levels of total amino acids, lactate, and total glutathione in comparison to normal tissues, and had a significantly lower ATP/AMP ratio (Supplementary Fig. 2).

\section{Tumor tissues with or without peritoneal recurrence}

We then performed a PLS analysis to characterize the metabolites that potentially associated with the presence or absence of peritoneal recurrence (Fig. 2). As a result, tumor tissues with or without peritoneal recurrence were marginally separated along the PLS2 axis. PLS2 factor loading (Supplementary Table 2) revealed that in patients with peritoneal recurrence, the levels of several nucleoside monophosphates, including AMP, IMP, and GMP, tended to be lower, and that the levels of nucleosides such as adenosine and guanosine were higher in comparison to patients without peritoneal recurrence. The Mann-Whitney $U$ test revealed significant differences in the levels of 22 metabolites in tumor tissues from cases with or without peritoneal recurrence; this included nucleoside monophosphates AMP, GMP, and UMP, and their precursor amino acids Asp and Gly (Fig. 3). Interestingly, the levels of all of the metabolites were lower in patients with peritoneal recurrence than in those without, with the exception of adenosine.

We examined the potential associations of all 93 metabolites with several recurrence patterns. Four metabolites (Asp, $\beta$-Ala, GDP and Gly) showed significantly lower levels in patients with peritoneal recurrence than in those without by Bonferroni correction for multiple comparisons. (Fig. 4a).
Fig. 2 Partial least squares (PLS) revealed no difference between the tumor and normal tissues (red and blue marks, respectively), and some differences along the PLS2 axis between tumor tissues with and without peritoneal recurrence (yellow and green marks, respectively)

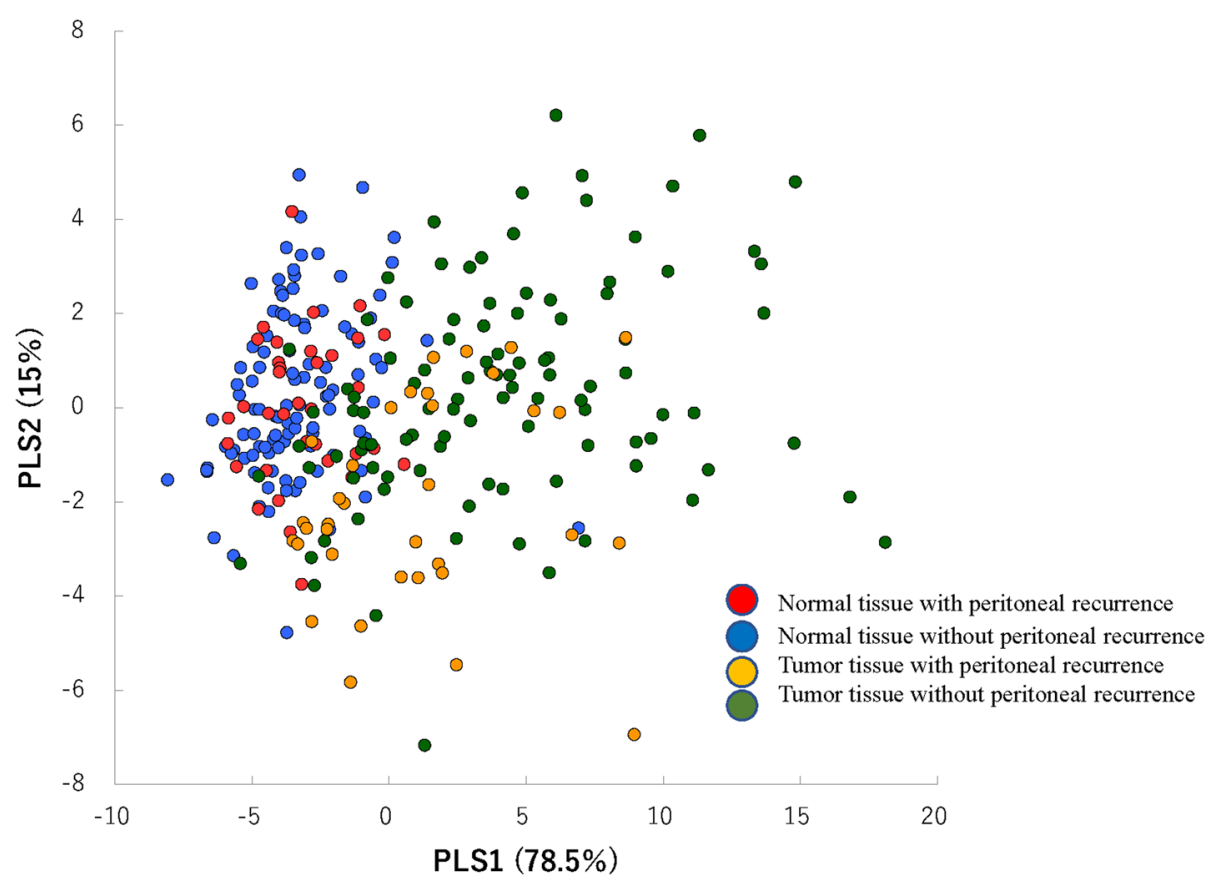




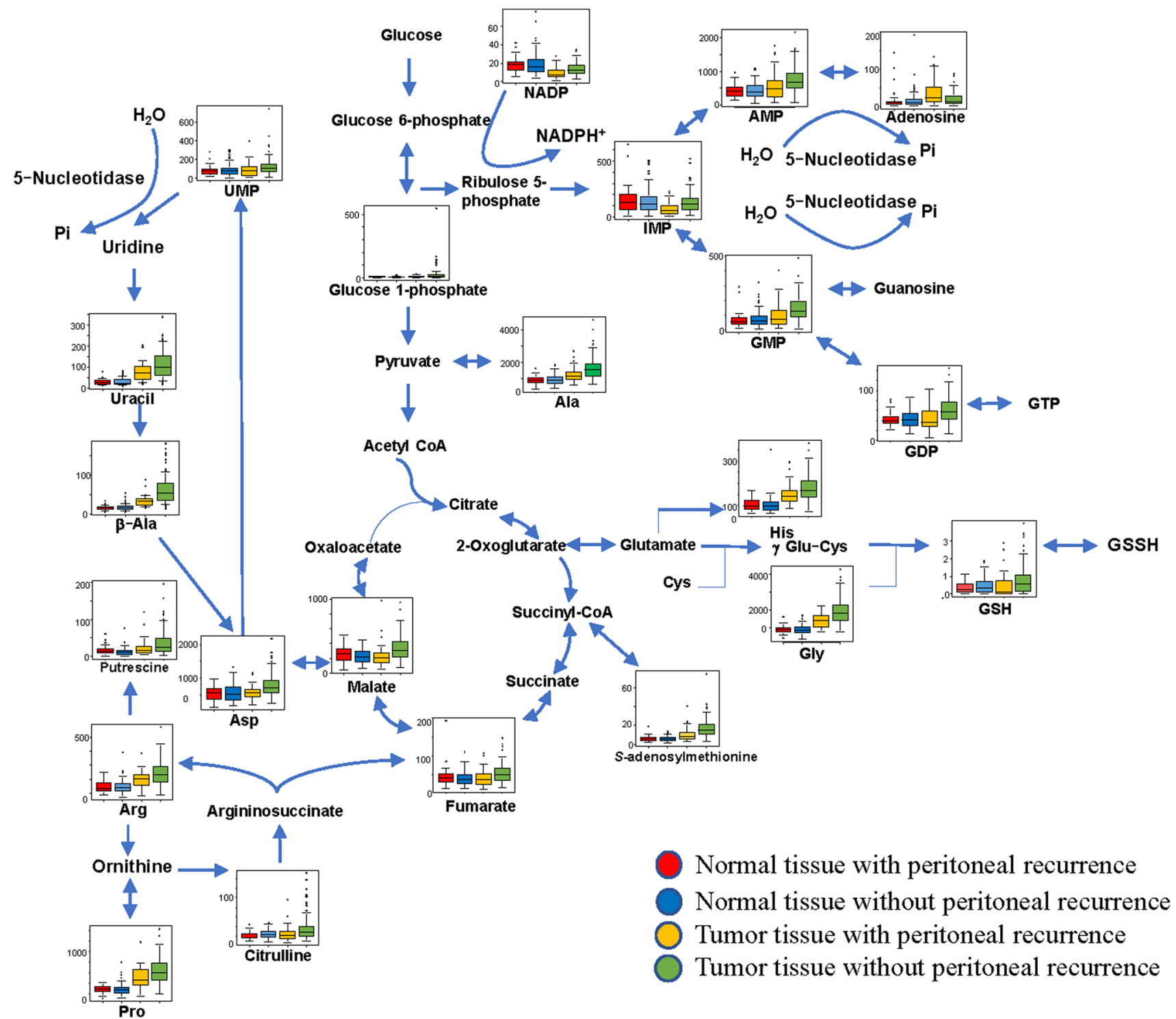

Fig. 3 Metabolomic date map of metabolites including amino acids and their derivatives in tumor tissues obtained from the patients with peritoneal recurrence or without peritoneal recurrence. Each bar indi-

However, there were no significant differences in the levels of any of the metabolites between patients with and without lymph-node or hematogenous recurrence (Fig. 4b, c). Based on the AUC of the ROC, the cut-off values of the four metabolites for predicting peritoneal recurrence were as follows: Asp, $601.2 \mathrm{nmol} / \mathrm{g}$, AUC 0.70; $\beta$-Ala, $49.5 \mathrm{nmol} / \mathrm{g}$, AUC 0.76 ; GDP, $36.5 \mathrm{nmol} / \mathrm{g}$, AUC 0.69; and Gly, $1392.5 \mathrm{nmol} / \mathrm{g}$, AUC 0.70) (Supplementary Fig. 3). To evaluate the predictive performance of the cut-off values, we conducted eightfold cross-validation in this cohort. A multivariable logistic regression analysis revealed that lower $\beta$-Ala levels in tumor tissue and a deeper tumor depth were independent predictors of peritoneal recurrence (Table 1). cates the median concentration (nmol/g tissue) of tumor tissues. All the $p$ values were evaluated by the Mann-Whitney $U$ test using calculating $q$ value as False Discovery Rate $(p<0.05, q<0.05)$

\section{Relapse-free survival and multivariable logistic regression}

The 3-year relapse-free survival rates of the two groups, which were categorized based on the cut-off values for each of the four metabolites, are shown in Supplementary Fig. 4. Patients whose Asp, $\beta$-Ala, GDP, and Gly levels were higher than the cut-off values showed significantly longer 3-year relapse-free survival. As with relapse-free survival, patients in whom the levels of the four metabolites were lower than the cut-off values showed significantly poor 5 -year overall survival (Fig. 5a-d). In addition, a multivariable analysis revealed that the lower levels of $\beta$-Ala in tumor tissues, 
Fig. 4 a-c Metabolomic profiles of tumor tissues with or without peritoneal recurrence (a), hematogenous recurrence $(\mathbf{b})$, and lymph-node recurrence (c). In patients with peritoneal recurrence, the levels of 4 metabolites were significantly lower than those in patients without peritoneal recurrence a ASP $n$ mol/g



recurrence status

GDP

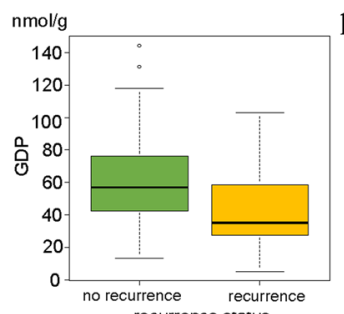

recurrence status

b ASP nmol/g

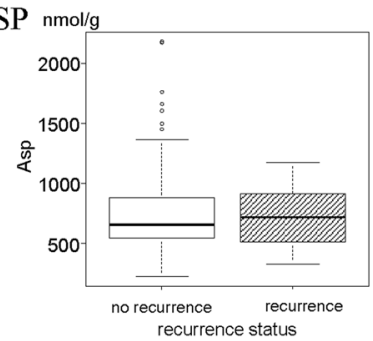

GDP

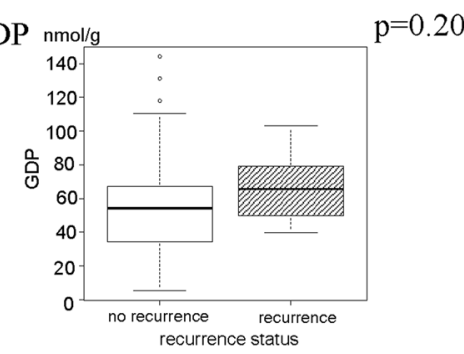

c ASP $n m o l / g$

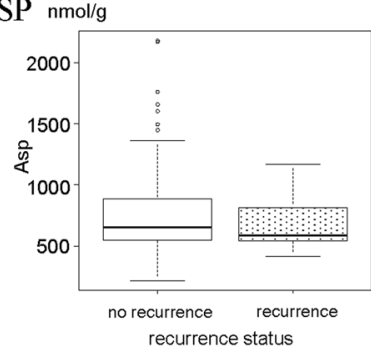

\section{GDP}



$p=0.97$ $\mathrm{p}<0.001 \beta \mathrm{Ala}$

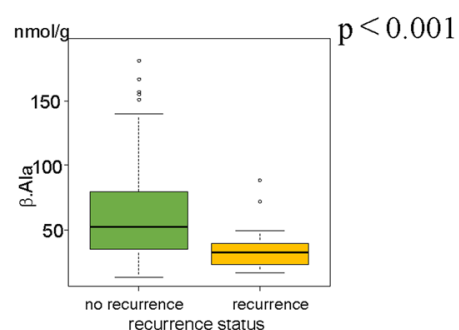

$\mathrm{p}=0.002$ Gly

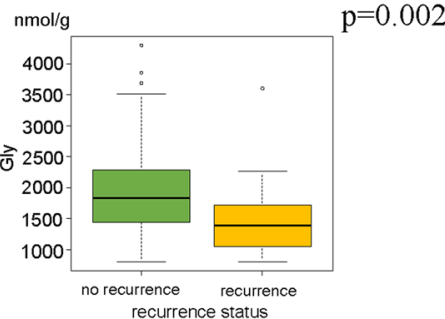

$p=0.86$

$\beta$ Ala nmolg

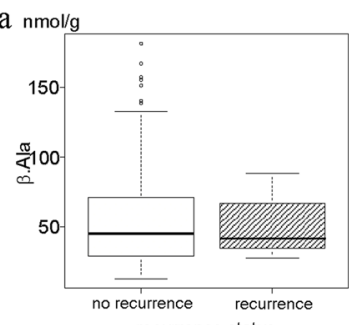

$p=0.94$

Gly

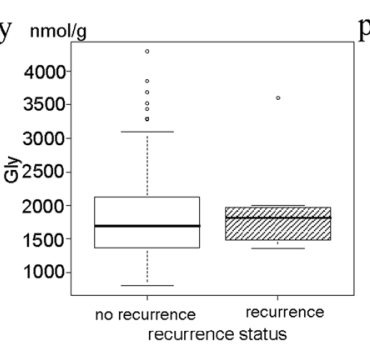

$p=0.63$

$p=0.55$

$\beta$ Ala nmol/g

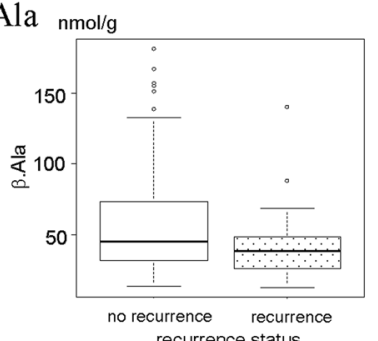

$p=0.26$
Gly

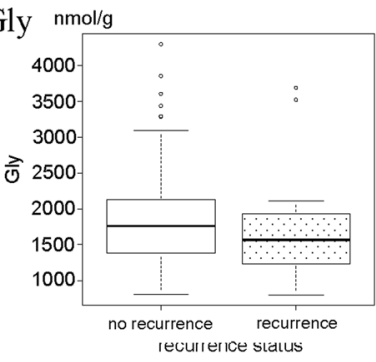


Table 1 Results of the multivariate logistic regression analysis of factors associated with peritoneal recurrence

\begin{tabular}{|c|c|c|c|c|c|c|}
\hline \multirow[t]{2}{*}{ Risk factors } & \multicolumn{3}{|c|}{ Univariate analysis } & \multicolumn{3}{|c|}{ Multivariate analysis } \\
\hline & OR & $95 \% \mathrm{CI}$ & $p$ & OR & $95 \% \mathrm{CI}$ & $p$ \\
\hline Sex (male/female) & 1.05 & $0.39-3.01$ & 0.91 & & & \\
\hline Age $(\geq 65 /<65$ years $)$ & 0.63 & $0.32-1.22$ & 0.11 & & & \\
\hline Tumor depth (pT1, 2, 3 vs. pT4) & 13.16 & $3.78-74.04$ & $<0.001^{*}$ & 6.37 & $1.40-42.60$ & $0.011^{*}$ \\
\hline Lymph-node metastasis (pN0 vs. pN1, 2, 3) & 14.90 & $2.50-502.1$ & $0.009^{*}$ & 6.82 & $0.77-262.84$ & 0.097 \\
\hline Histological type (differentiated type ${ }^{a}$ ) & 0.27 & $0.09-0.72$ & $0.004^{*}$ & 0.80 & $0.18-3.42$ & 0.738 \\
\hline CY (positive/negative) & 5.47 & $2.03-15.17$ & $<0.001^{*}$ & 1.64 & $0.46-5.79$ & 0.370 \\
\hline$\beta$ Ala $(<49.5 / \geq 49.5 \mathrm{nmol} / \mathrm{g})$ & 12.19 & $3.51-68.55$ & $<0.001^{*}$ & 5.21 & $1.07-35.89$ & $0.029 *$ \\
\hline $\operatorname{ASP}(<601.2 / \geq 601.2 \mathrm{nmol} / \mathrm{g})$ & 4.37 & $1.76-11.48$ & $<0.001^{*}$ & 2.31 & $0.67-8.13$ & 0.125 \\
\hline $\operatorname{GDP}(<36.5 / \geq 36.5 \mathrm{nmol} / \mathrm{g})$ & 6.14 & $2.37-16.56$ & $<0.001^{*}$ & 1.95 & $0.49-7.83$ & 0.270 \\
\hline Gly $(<1392.5 / \geq 1392.5 \mathrm{nmol} / \mathrm{g})$ & 4.50 & $1.76-11.74$ & $<0.001 *$ & 1.19 & $0.29-4.69$ & 0.766 \\
\hline
\end{tabular}

$O R$ odds ratio, $C I$ confidence interval

* Statistically significant

${ }^{a}$ Differentiated type: moderately differentiated and/or well-differentiated adenocarcinoma, and/or papillary adenocarcinoma
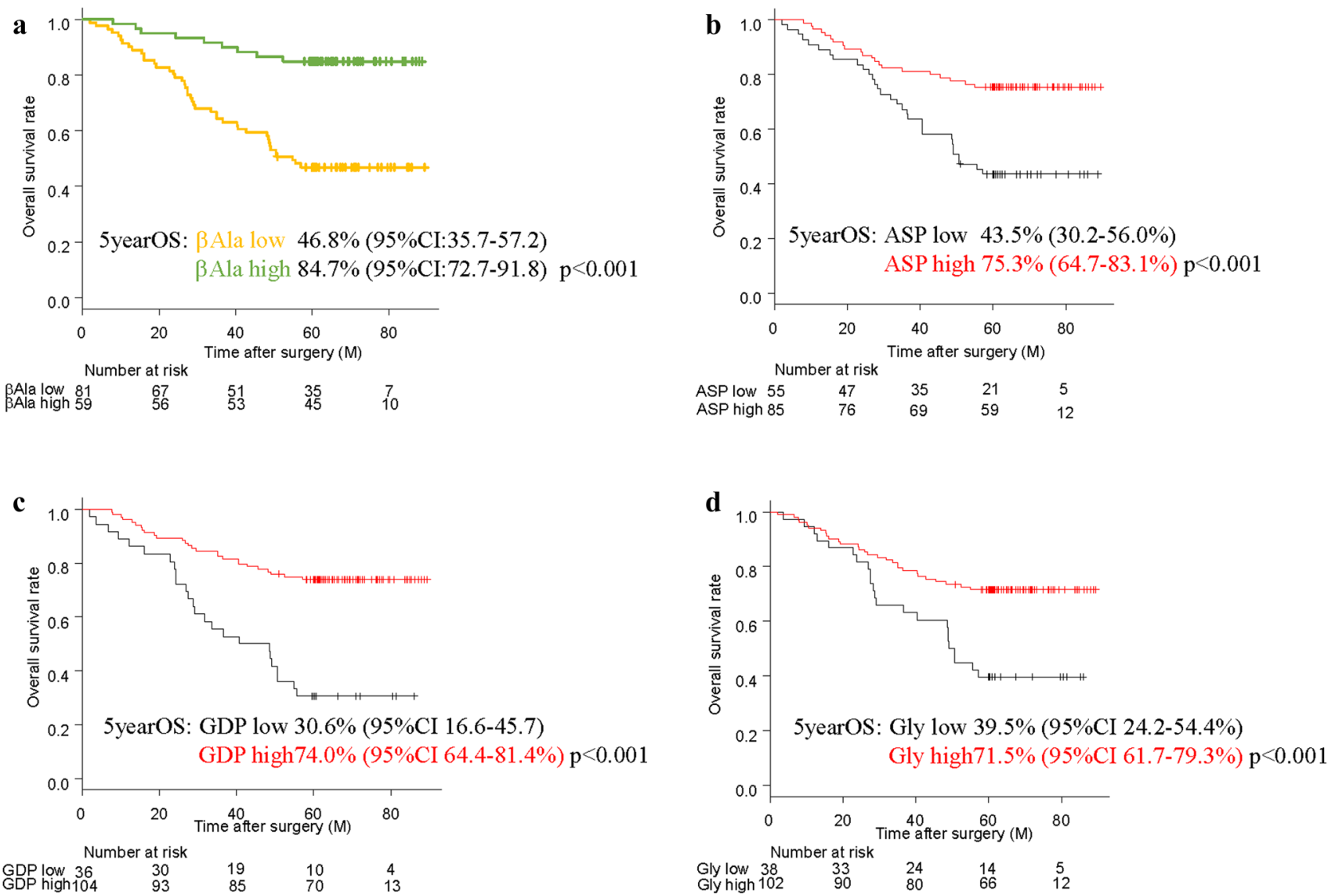

Fig. 5 a-d Five-year overall survival rates of patients with high and low levels of each of the four metabolites. The patients were categorized into high and low groups based on the cut-off values for $\beta$-Ala ASP, GDP and Gly 
positive lymph-node metastasis, and positive washing peritoneal cytology were independent predictors of overall survival (Supplementary Table 3).

\section{Transwell migration assay}

We conducted a Transwell migration assay using a poorly differentiated gastric cancer cell line (TMK1). The number of migrated cells was significantly reduced by $48 \mathrm{~h}$ of treatment with $\beta$-Ala (Fig. 6).

\section{Discussion}

It is widely known that cancer cells rely more on anaerobic glycolysis than oxidative phosphorylation to produce energy (Warburg effect) [22]. However, the associations of tissue metabolite patterns with the likelihood of developing peritoneal recurrence have rarely been studied. In this study, we analyzed the metabolite levels of gastric cancer tissues from 140 patients who underwent gastrectomy and confirmed that there were dramatic differences between tumor and normal tissues. We then clarified the metabolites that were mainly associated with peritoneal recurrence by PLS and a multivariable logistic regression analysis of the metabolite levels in patients with and without peritoneal recurrence. In particular, $\beta$-Ala had a strong correlation with both peritoneal recurrence and overall survival.

$\beta$-Ala is a degradation product of dihydrouracil, and an intermediate metabolite of L-carnosine synthesis. While the anti-tumor effects of L-carnosine have been extensively studied [23], recent studies have suggested the effects of $\beta$-Ala itself on cancer proliferation and metastasis. For example, Vaughan et al. suggested that $\beta$-Ala reduced both cell migration and the proliferation of malignant breast epithelial cells through alterations in metabolism and cellular acidity [24]. $\beta$-Ala also showed various anti-tumor effects, including the suppression of invasion and migration in metastatic human osteosarcoma cells [25]. Indeed, an in vitro experiment indicated that treatment with a subtoxic concentration of $\beta$-Ala suppressed the migration of the TMK1 cells (a poorly differentiated gastric cancer cell line) in a Transwell migration assay. Thus, the decrease of $\beta$-Ala levels may have exacerbated the metastaticity of the residual gastric cancer cells, leading to the increased frequency of peritoneal recurrence. $\beta$-Ala is available on the market as a dietary supplement that may help exercise performance without serious side effects. There is a possibility that dietary supplementation of $\beta$-Ala may prevent peritoneal recurrence in gastric cancer patients and lead to the amelioration of overall survival. Further studies are required to understand the molecular mechanisms of the anti-cancer effects of $\beta$-Ala, how $\beta$-Ala is supplied from the tumor microenvironment, and which cells (e.g., cancer cells, cancer-associated fibroblasts, and tumor-associated macrophages) mainly produce this metabolite.

The PLS analysis also revealed that nucleoside monophosphate levels were lower in cancer with peritoneal recurrence, whereas the levels of nucleosides, such as adenosine, were higher (Fig. 3, Supplementary Table 2). Many types of cancer, including gastric cancer, have been shown to highly express NT5E (5'-nucleotidase ecto; also known as

a



TMK 1-control

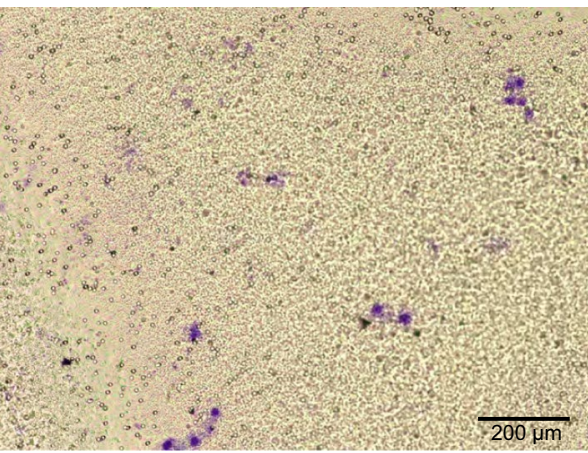

TMK 1-ß-Ala

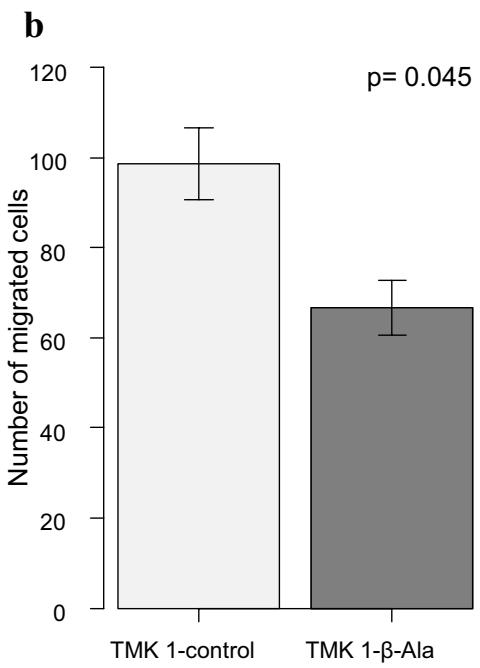

Fig. 6 a, b A Transwell migration assay using TMK1 cells. a A microscopic image of migrated TMK1 cells incubated with or without $40 \mathrm{mM} \beta$-Ala for $48 \mathrm{~h}$. b A Transwell migration assay using TMK1 cells. In cells incubated with $40 \mathrm{mM} \beta$-Ala, the numbers of migrated cells were significantly lower in comparison to cells incubated without $\beta$-Ala. The data represent the mean \pm SEM from three independent experiments. A paired two-tailed $t$ test was used to calculate statistical significance 
CD73), which converts extracellular nucleoside monophosphates to nucleosides (e.g., AMP to adenosine) [26]. Our data suggest that the gastric cancer tissues with peritoneal recurrence had even higher NT5E expression levels. Previous studies have suggested that the adenosine produced by NT5E on the surface of cancer cells activates the adenosine receptors expressed in immune cells such as regulatory $\mathrm{T}$ cells and leads to immunosuppression [27]. Adenosine receptors are also expressed in cancer cells and promote their growth and migration $[28,29]$. Thus, it is possible that increased levels of nucleosides, especially adenosine, may have contributed to the poor prognosis of these patients.

In conclusion, we performed a metabolomics analysis on gastric cancer tissues and identified several metabolic changes that were potentially related to peritoneal recurrence. In particular, $\beta$-Ala may serve as an independent predictor of peritoneal recurrence and an independent prognostic factor for overall survival. Further studies of other cohorts should be performed to validate our findings and the potency of metabolites as biomarkers for predicting peritoneal recurrence.

Acknowledgements We thank Yoshihiro Mezawa and Kazunori Kajino for their technical assistance in the migration assay.

Author contribution Study concept and design: SK, TI, MK, KK, YO, and TM; acquisition of data: SK, RM, YY, MT, YT, EB, TK, and TM; analysis/interpretation of data: SK, TI, KK, YO, and MT; drafting of the manuscript: MK, MT, and MT; critical revision of manuscript: KK, H-CL-O, and MT; technical, or material support: MK, KK, YO, and MT; experimental assistance: SZ, OH H-CL-O, and FT; final approval of manuscript: all authors.

Funding Study funding was provided by Shizuoka Cancer Center.

Data availability The corresponding author has access to all data in the study.

\section{Compliance with ethical standards}

Conflict of interest The authors declare that they have no conflicts of interest.

Ethics approval This study was approved by the Institutional Review Board of Shizuoka Cancer Center. The study was performed in accordance with the Declaration of Helsinki.

Informed consent Written informed consent for the use of tissue samples and data were obtained from all patients.

\section{References}

1. Allemani C, Weir HK, Carreira H, Harewood R, Spika D, Wang X-S, et al. Global surveillance of cancer survival 1995-2009: analysis of individual data for 25676887 patients from 279 population-based registries in 67 countries (CONCORD-2). The Lancet. 2015;385(9972):977-1010.
2. Takahashi T, Saikawa Y, Kitagawa Y. Gastric cancer: current status of diagnosis and treatment. Cancers (Basel). 2013;5(1):48-63.

3. Shiozaki H, Elimova E, Slack RS, Chen HC, Staerkel GA, Sneige N, et al. Prognosis of gastric adenocarcinoma patients with various burdens of peritoneal metastases. J Surg Oncol. 2016;113(1):29-35

4. Katai H, Ishikawa T, Akazawa K, Isobe Y, Miyashiro I, Oda I, et al. Five-year survival analysis of surgically resected gastric cancer cases in Japan: a retrospective analysis of more than 100,000 patients from the nationwide registry of the Japanese Gastric Cancer Association (2001-2007). Gastric Cancer. 2018;21(1):144-54.

5. Van Cutsem E, Sagaert X, Topal B, Haustermans K, Prenen H. Gastric cancer. The Lancet. 2016;388(10060):2654-64.

6. Miki Y, Tokunaga M, Tanizawa Y, Bando E, Kawamura T, Terashima M. Staging Laparoscopy for Patients with cM0, Type 4, and Large Type 3 Gastric Cancer. World J Surg. 2015;39(11):2742-7.

7. Mayers JR, Wu C, Clish CB, Kraft P, Torrence ME, Fiske BP, et al. Elevation of circulating branched-chain amino acids is an early event in human pancreatic adenocarcinoma development. Nat Med. 2014;20(10):1193-8.

8. Battini S, Faitot F, Imperiale A, Cicek AE, Heimburger C, Averous $\mathrm{G}$, et al. Metabolomics approaches in pancreatic adenocarcinoma: tumor metabolism profiling predicts clinical outcome of patients. BMC Med. 2017;15(1):56.

9. Farid SG, Morris-Stiff G. "OMICS" technologies and their role in foregut primary malignancies. Curr Probl Surg. 2015;52(10):409-41.

10. Abbassi-Ghadi N, Kumar S, Huang J, Goldin R, Takats Z, Hanna GB. Metabolomic profiling of oesophago-gastric cancer: a systematic review. Eur J Cancer. 2013;49(17):3625-37.

11. Hirayama A, Kami K, Sugimoto M, Sugawara M, Toki N, Onozuka H, et al. Quantitative metabolome profiling of colon and stomach cancer microenvironment by capillary electrophoresis time-of-flight mass spectrometry. Cancer Res. 2009;69(11):4918-25.

12. Hur H, Paik MJ, Xuan Y, Nguyen DT, Ham IH, Yun J, et al. Quantitative measurement of organic acids in tissues from gastric cancer patients indicates increased glucose metabolism in gastric cancer. PLoS ONE. 2014;9(6):e98581.

13. Song H, Wang L, Liu HL, Wu XB, Wang HS, Liu ZH, et al. Tissue metabolomic fingerprinting reveals metabolic disorders associated with human gastric cancer morbidity. Oncol Rep. 2011;26(2):431-8.

14. Wu H, Xue R, Tang Z, Deng C, Liu T, Zeng H, et al. Metabolomic investigation of gastric cancer tissue using gas chromatography/ mass spectrometry. Anal Bioanal Chem. 2009;396(4):1385-95.

15. Yuan LW, Yamashita H, Seto Y. Glucose metabolism in gastric cancer: The cutting-edge. World J Gastroenterol. 2016;22(6):2046-59.

16. Chen XD, Mao CC, Wu RS, Zhang WT, Lin J, Sun XW, et al. Use of the combination of the preoperative platelet-to-lymphocyte ratio and tumor characteristics to predict peritoneal metastasis in patients with gastric cancer. PLoS ONE. 2017;12(4):e0175074.

17. Association JGC. Japanese classification of gastric carcinoma. 15th ed. Tokyo: Kanehara Publisher; 2017.

18. Ohashi T, Komatsu S, Ichikawa D, Kosuga T, Okamoto K, Arita $\mathrm{T}$, et al. Monitoring with sensitive tumor markers contributes to decision-making and better prognosis in gastric cancer patients with peritoneal recurrence. Int J Clin Oncol. 2017;22(5):897-904.

19. Ooga T, Sato H, Nagashima A, Sasaki K, Tomita M, Soga T, et al. Metabolomic anatomy of an animal model revealing homeostatic imbalances in dyslipidaemia. Mol BioSyst. 2011;7(4):1217-23.

20. Sugimoto M, Wong DT, Hirayama A, Soga T, Tomita M. Capillary electrophoresis mass spectrometry-based saliva metabolomics 
identified oral, breast and pancreatic cancer-specific profiles. Metabolomics. 2010;6(1):78-95.

21. Yamamoto H, Fujimori T, Sato H, Ishikawa G, Kami K, Ohashi Y. Statistical hypothesis testing of factor loading in principal component analysis and its application to metabolite set enrichment analysis. BMC Bioinform. 2014;15:51.

22. Vander Heiden MG, Cantley LC, Thompson CB. Understanding the Warburg effect: the metabolic requirements of cell proliferation. Science (New York, NY). 2009;324(5930):1029-33.

23. Iovine B, Iannella ML, Nocella F, Pricolo MR, Bevilacqua MA. Carnosine inhibits KRAS-mediated HCT116 proliferation by affecting ATP and ROS production. Cancer Lett. 2012;315(2):122-8.

24. Vaughan RA, Gannon NP, Garcia-Smith R, Licon-Munoz Y, Barberena MA, Bisoffi M, et al. beta-alanine suppresses malignant breast epithelial cell aggressiveness through alterations in metabolism and cellular acidity in vitro. Mol Cancer. 2014;13:14.

25. Li S, Chen P, Zheng K, Wang W, Pei Y, Qiu E, et al. beta-Alanine mediated inhibition of PTHR1suppresses the proliferation, invasion and tumorigenesis in metastatic human osteosarcoma U2OS cells. Int J Biol Macromol. 2018;111:1255-63.
26. Hu S, Meng F, Yin X, Cao C, Zhang G. NT5E is associated with unfavorable prognosis and regulates cell proliferation and motility in gastric cancer. Biosci Rep. 2019. https://doi.org/10.1042/ BSR20190101.

27. Allard B, Longhi MS, Robson SC, Stagg J. The ectonucleotidases CD39 and CD73: novel checkpoint inhibitor targets. Immunol Rev. 2017;276(1):121-44.

28. Cappellari AR, Rockenbach L, Dietrich F, Clarimundo V, Glaser $\mathrm{T}$, Braganhol E, et al. Characterization of ectonucleotidases in human medulloblastoma cell lines: ecto-5'NT/CD73 in metastasis as potential prognostic factor. PLoS ONE. 2012;7(10):e47468.

29. Yu J, Wang X, Lu Q, Wang J, Li L, Liao X, et al. Extracellular 5'-nucleotidase (CD73) promotes human breast cancer cells growth through AKT/GSK-3beta/beta-catenin/cyclinD1 signaling pathway. Int J Cancer. 2018;142(5):959-67.

Publisher's Note Springer Nature remains neutral with regard to jurisdictional claims in published maps and institutional affiliations. 